\title{
Is Hemifacial Spasm of Peripheral or Central Origin?
}

Hemifacial spasm (HFS) is a problematical condition for the affected individual and a puzzling one for the clinician. The movement severity and extent progress with time leading many sufferers to seek a surgical intervention. With the theory that the HFS is due to the pulsatile action of a blood vessel on the nerve, the surgical separation of the two structures has been postulated as the best approach, and it is one that is successful in many patients. However, the origin of the condition remains elusive. Is it due to just the repeated pulsatile flow on a nerve, or is there a more central origin? In this issue Wilkinson and Kaufmann present new data indicative of a central origin. ${ }^{1}$ Their approach is best on intraoperative recording of motor evoked potentials in the facial muscles. Providing care is taken to ensure that these responses are truly central in origin and do not arise from extracranial activation of the nerve, these are robust measures of cranial nerve function ${ }^{2,3}$. They compare the latency and amplitude of the potentials between HFS patients and those coming for other skull base surgeries. Showing no difference between these two, they conclude that there is little, if any, peripheral nerve damage in the skull-base surgery patients and that comparisons of other measures are reasonable. Broadly, this seems reasonable, but there may of course be other issues affecting the skull-base surgery patients which may alter the motor evoked potential (MEP) properties.

The Winnipeg group then show that the threshold for eliciting the MEP with multi-pulse stimulation, the standard approach in the operating room, is lower in the HFS patients than in the skull-base surgery patients. ${ }^{1}$ The difference is remarkably large, and care was taken that amplitudes between the two groups were similar. This difference is ascribed to a variation in the excitability of the motor neuron. The motor evoked potential is not adept at identifying differences in excitability of a given cell along the pathway of study and so either the cell in the cortex or pons may be hyper-excitable.

When subjects are awake it is usual for the motor evoked potential to be elicited by a single pulse of stimulation, either delivered through a magnet or electrically. When generated magnetically many waves of stimulation are transmitted along the spinal cord, but single pulse transcranial electrical stimulation (TES) in the awake subject generates a single direct (D)-wave ${ }^{4}$ which can result in an MEP. Under anesthesia although we know that D-waves are generated they are usually not sufficient to record a MEP in a muscle, at least in healthy subjects. The likelihood of a hyper-excitable motor neuron as a generator for HFS is strengthened therefore by the observation that single pulse TES under anesthesia generated MEPs at similar stimulation voltages, and with similar latencies and amplitudes to those seen with multi-pulse stimulation.
Of course, with a study involving patients undergoing surgery there are selection bias effects to be taken into account. However with the ease of obtaining MEPs in the awake subject it is surely only a matter of time before this approach is used in pre-surgical patients. During surgery the separation of the blood vessel from the nerve did remove the lateral spread response. Did this persistent beating on the nerve generate the hyper-excitability, or do you need both a hyper-excitable neuron and a blood vessel touching the nerve?

Jonathan Norton

Department of Surgery, University of Saskatchewan Saskatoon, Saskatchewan, Canada Email:j.norton@usask.ca

\section{REFERENCES}

1. Wilkinson MF, Kaufmann AM. Facial motor neuron excitability in hemifacial spasm: a facial MEP study. Can J Neurol Sci. 2014; 41(2):239-45.

2. Edwards B, Kileny P. Intraoperative neurophysiologic monitoring: indications and techniques for common procedures in otolaryngology-head and neck surgery. Otolaryngol Clin North Am. 2005;38(4):631-42.

3. Nuwer M. Intraoperative monitoring of neural function. Vol 8. New York: Elsevier; 2008.

4. Day B, Dressler D, Maertens de Noordhout A, et al. Electric and magnetic stimulation of human motor cortex: surface EMG and single motor unit responses. J Physiol. 1989;412:449-73. 